\title{
Assessment of Physical and Mental Health in Male University Students with Varying Sleep Habits
}

\author{
YUUKI MATSUMOTO, KOUJI TOYOMASU* AND NAOHISA UCHIMURA** \\ Kurume University Graduate School of Medicine, *Institute of Health and Sports Science and \\ **Department of Neuropsychiatry, Kurume University School of Medicine, \\ Kurume 830-0011, Japan
}

Received 28 November 2011, accepted 24 January 2012

Edited by TATSUYA ISHITAKE

\begin{abstract}
Summary: Healthy sleep habits entail not only sleeping for a sufficient period (quantity) but also regularity of the sleep cycle and getting sound sleep (quality). University students often have erratic schedules that cause irregular sleep patterns even though sleep durations remain relatively constant. This study compared the physical and mental health of 90 male university students with different sleep habits. We created sleep habit scales using the Tokyo Metropolitan Institute for Neuroscience life habits inventory (TMIN-LHI; Miyashita, 1994) by performing a factor analysis and classifying sleeping habits based on regularity, quality, and quantity. Four types of sleep habits were identified by cluster analysis; good sleep was characterized by regular and high quality sleep but of relatively short sleep duration; long sleep was regular and relatively long but of low quality; short sleep was of high quality but short and irregular, while poor sleep was irregular, of low quality, and relatively long. The good sleep group had a significantly lower average waist circumference, and lower systolic and diastolic blood pressure. The long and poor sleep groups, which both had low quality sleep, scored lower than the national standard on the mental component summary (MCS) calculated from the Social Functioning-36 (SF-36) short-form health survey. Furthermore, the average MCS score of the poor sleep group was significantly lower than that of any other sleep habit group. Subjects with poor sleep also scored lowest on the Self-rating Depression Scale (SDS). In addition, the short and poor sleep groups were prone to glucose or lipid metabolism disorders. Maintaining good physical and mental health without sound sleep and a regular sleep cycle is difficult, even if sleeping hours are kept constant. Therefore, we included the assessment of regularity and quality in addition to hours of sleep in order to develop appropriate sleep guidelines for improved physical and mental health.
\end{abstract}

Key words university students, sleep habits, sleep hygiene, regularity of sleep, quality of sleep, sleep duration

\section{INTRODUCTION}

Metabolic syndrome and depression are now attracting considerable research attention. These disorders are closely related to various aspects of lifestyle, such as diet [1], exercise [2], and mental stress [3]. The Japanese population has a tendency to neglect sleep because of a national work ethic based on diligence [4]. In addition, working overtime is considered a virtue in Japan, and 24-h work operations are commonplace. Twenty-four hour stores are a regular feature and many young people work in late shifts. For them, work time and free time are more important than sleep duration [5].

Correspondence: Yuuki Matsumoto, Department of Health Science, Kurume University Graduate School of Medicine, Kurume 830-0011, Japan. Tel: 0909567-3709 Fax: 0942-39-1637 E-mail: aruma_tio@hotmail.com

Abbreviations: ANOVA, analysis of variance; ASDA, American Sleep Disorders Association; BMI, body mass index; FBS, fasting blood sugar; HDL-C, high-density cholesterol; HMW-adiponectin, high molecular weight adiponectin; HOMA-IR, homeostasis model of assessment-insulin resistance; HSD, honestly significant difference; LDL-C, low-density cholesterol; ME, Morningness-Eveningness; MCS, mental component summary; n.s., not significant; PCS, physical component summary; QOL, quality of life; SD, standard deviation; SDS, Self-rating Depression Scale; SF-36, Social Functioning-36; TMINLHI, Tokyo Metropolitan Institute for Neuroscience life habits inventory. 
However, irregular and insufficient sleep can cause daytime drowsiness and a lack of concentration, reduced quality of life, and a decline in performance. For example, Belenky [6] has indicated that once chronic sleep insufficiency affects performance, complete recovery is difficult even if adequate sleep was secured for three consecutive nights. When chronic sleep insufficiency gradually accumulates in an individual, performance is drastically reduced even if sleepiness is not felt. Lack of sleep and insomnia also cause mental disorders. Kaneita's study [7] on 24,686 Japanese adults ascertained that sleep duration of less than six $h$ and more than eight $h$ was associated with symptoms of depression. Sleep deficiency can affect physical health as well. Gangwish [8] found that subjects who slept less than four hours were $73 \%$ more obese than those who slept more than seven h. Tochikubo [9] reported a high blood pressure throughout the day in subjects who had only 3-4 h of sleep the previous night. Knutsona [10] demonstrated that one week of sleep insufficiency caused impaired glucose tolerance because of a decline in insulin sensitivity levels. Therefore, adequate sleep is important not only for performing normal daytime activities efficiently, but also for good physical and mental health.

University students often have erratic schedules that cause irregular sleep patterns. Kang [11] suggested that students with an irregular bedtime schedule might experience poor sleep quality. In addition, Buboltz [12] investigated that poor sleep habits might become a self-perpetuating cycle that students are unaware of and might be unable to alter. Chang [13] conducted a follow-up study on 1053 male university graduates for 34 years. Of these, 103 developed depression, and the risk of depression in those who had insomnia during their university days was twice as high as that in subjects who did not. Therefore, it has been suggested that poor sleep habits at a young age could have an effect on sleep habits in middle age in the same individual, and that sleep habits are not shortterm but rather long-term factors.

When evaluating sleep, we tend to emphasize the number of hours (i.e., the quantity of sleep). However, maintaining a regular and sound sleep cycle are also important factors. Hayashi [14] stated that sleep habits could be measured in three dimensions (i.e., regularity, quality and quantity). In an earlier study, Takeuchi [15] identified these three factors of sleep from the Tokyo Metropolitan Institute for Neuroscience life habits inventory (TMIN-LHI) and classified sleep habits on the basis of these factors. Takeuchi's analysis takes into account not only the quantity of sleep but also examines other perspectives, by which the issue can be more comprehensively understood. Minimizing hidden perspectives in this way provided a better understanding of the sleep habits of university students. However, no physical or mental data were investigated in that study, so the relationship between health condition and sleep habits was ambiguous. University students often have physical and mental problems besides sleep disorders [16]. In this study, we used Takeuchi's method to compare the physical and mental condition of university students in order to clarify the effects of certain factors that cause differences in sleep habits. In addition, because sleep habits differ between sexes [17], we restricted our study to males in order to obtain a more exact analysis. Therefore, we investigated sleep habits (sleep hygiene) in male university students for the purpose of demonstrating the association between sleep habits and physical and mental health.

\section{MATERIALS AND METHODS}

\section{Study subjects and duration}

The subjects of this study were male students of Kurume University (18-29 years; average age: mean \pm standard deviation (SD), $19.4 \pm 1.8$ years). None of the subjects had any physical or mental disease and none were on regular medication. In addition, obesity can cause secondary sleep disturbances because of sleep apnea syndrome. Therefore, a body mass index (BMI) of 18.5 to 24.9 was set as an inclusion factor for this study. In total, 90 students were included in this study, which was carried out in June 2010. There were no aggravating circumstances such as examinations or long breaks before or after the testing day.

\section{Questionnaires}

We used the TMIN-LHI, which is a detailed questionnaire comprising two sections. The first section includes 60 questions based on sleep habits and other lifestyle issues. The second section is a MorningnessEveningness (ME) questionnaire created by Horne and Ostberg [18] and translated into Japanese by Ishihara [19]. It comprises 19 items. We calculated the ME score for our subjects on the basis of their grade slips.

\section{Preparation of the sleep habits scale and classification of subjects}

\section{Selected Items}

From TMIN-LHI, 26 of 60 items from section 1 were excluded. These were nominal items or the items targeting certain people who offered a specific answer, and had large ceiling or floor effects. We eventually 
conducted a factor analysis with 35 items (i.e., the remaining 34 items from section 1 and the ME score from section 2).

\section{Factor Analysis}

We extracted factors by principal factor analysis and ran a promax rotation. Three factors were chosen on the basis of scree plot. The characteristic values were $6.120,3.030$ and 2.591 respectively, and the cumulative percentage was $33.5 \%$.

Following analysis of the 35 items from the TMINLHI, which was done five times, 18 items were excluded. The remaining 17 items had factor loadings of $\geqq 0.35$. The explanatory power was $53.4 \%$ and the accumulated contribution rate was $46.0 \%$. The confidence coefficients $(\alpha)$ of the three factors were 0.878 , 0.683 , and 0.670 for the first, second and third factor, respectively.

The three factors focused on in this study are described in Table 1 along with their factor loadings. The first factor comprised 8 items: regular bedtime, regular wake-up time, irregular bedtime, irregular wake-up time, ME score, irregular sleep duration, breakfast habits, and exercise habits. This regularity factor along with its items was named the related Sleep Regularity scale. The second factor comprised 5 items: difficulty of sleep latency, time to fall asleep, mood on waking up in the morning, depth of sleep and experience of insomnia. This quality factor along with its items was named the related Sleep Quality scale. The third factor comprised 5 items: regular sleep duration, ideal sleep duration, regular wake-up time, value attached to sleep and time spent in commuting. This quantity factor along with its items was named the related Sleep Quantity scale.

\section{Principal Component Analysis}

Eight items from the first factor, five from the second factor and five from the third factor were subjected to principal component analysis, and each factor was given a standard score. We created a sleep habits scale by assuming the first score to be a scale score. The average score was initially fixed at 0 . In the related Sleep Regularity scale, a positive score indicated mostly regular sleep habits and a negative score indicated mostly irregular sleep habits. In the related Sleep Quality scale, a positive score indicated a more sound sleep and a negative score indicated a very disturbed sleep. In the related Sleep Quantity scale, a positive score indicated longer sleep duration and more active procurement of sleep, and a negative score indicated shorter sleeping hours and more passive procurement of sleep. Classifying Subjects

The Ward method of cluster analysis was selected

TABLE 1.

Extracted factors and factor loadings

\begin{tabular}{|c|c|c|c|}
\hline & Items & Range & Factor Loadings \\
\hline \multirow{8}{*}{$\begin{array}{l}\text { The First } \\
\text { Factor } \\
\alpha=0.878\end{array}$} & Q1: regular bedtime & early - late & 0.868 \\
\hline & Q4: regular wake-up time & early - late & 0.745 \\
\hline & Q2: irregular bedtime & small - large & 0.713 \\
\hline & Q5: irregular wake-up time & small - large & 0.689 \\
\hline & ME score & Eveningness - Morningness & -0.657 \\
\hline & Q8: irregular sleep duration & small - large & 0.652 \\
\hline & Q37a: breakfast habits & always - never & 0.616 \\
\hline & Q49: exercise habits & never - always & -0.596 \\
\hline \multirow{5}{*}{$\begin{array}{c}\text { The Second } \\
\text { Factor } \\
\alpha=0.683\end{array}$} & Q14: difficulty of sleep latency & easy - difficult & 0.982 \\
\hline & Q13: time to fall asleep & short - long & 0.529 \\
\hline & Q19: mood on waking up in the morning & pleasant - unpleasant & 0.427 \\
\hline & Q20: depth of sleep & deep - light & 0.417 \\
\hline & Q43: experience of insomnia & no - yes & 0.408 \\
\hline \multirow{5}{*}{$\begin{array}{l}\text { The Third } \\
\text { Factor } \\
\alpha=0.670\end{array}$} & Q7: regular sleep duration & short - long & 1.002 \\
\hline & Q11: ideal sleep duration & short - long & 0.574 \\
\hline & Q4: regular wake-up time & early - late & 0.397 \\
\hline & Q12: value attached to sleep & important - unimportant & -0.381 \\
\hline & Q45: time spent in commuting & short - long & -0.377 \\
\hline
\end{tabular}


for classifying the subjects on the basis of principal component score for each factor. Four clusters were determined because they were the most balanced and the clearest following the dendrogram.

\section{Physical factors}

The subjects were instructed to measure their own height and weight. Waist circumference was measured at the umbilical region by members of the same study staff. The subjects also measured their own resting blood pressure and pulse with an automatic manometer (BP-203RV Type C, Nippon Colin, Tokyo, Japan) in a sitting position. If systolic blood pressure was $\geqq 130 \mathrm{mmHg}$ or diastolic blood pressure was $\geqq 85$ $\mathrm{mmHg}$, subjects measured their blood pressure again. In the event that the second blood pressure measurement was unacceptable, the study staff measured it with a mercurial column manometer.

\section{Mental health}

We evaluated mental health using the Japanese version of the Social Functioning-36 (SF-36) [20-22] short-form health survey and Japanese version of the Self-rating Depression Scale (SDS) [23].

The Japanese version of the SF-36, which was developed by Fukuhara [20-22], is a general scale used to measure quality of life over a one-month period. All items are scaled, and the higher the scores, the better the quality of life. Physical component summary (PCS) and mental component summary (MCS) were derived from the SF-36: physical functioning, role physical, bodily pain, general health perceptions, vitality, social functioning, role emotional, and mental health. PCS score and MCS score can be compared to national standard values directly ( $50=$ national standard score). Furthermore, we can evaluate physical quality of life (QOL) from PCS and mental QOL from MCS in a comprehensive manner.

Zung $[24,25]$ developed SDS as simple test to assess depression, and Fukuda translated it into Japanese [23]. It comprises 20 items, all graded according to four ranks of 1-4 points each. We used an integrated scoring system as follows: $\leqq 39$ points indicated normal mental health, 40-49 points indicated slight depression, and $\geqq 50$ points indicated moderate depression.

\section{Blood tests}

Blood samples were taken and fasting blood sugar (FBS), immunoreactive insulin levels, high-density cholesterol (HDL-C), low-density cholesterol (LDL-C), leptin, des-acyl ghrelin, and high molecular weight adiponectin (HMW-adiponectin) were measured. In addition, the homeostasis model of assessment-insulin resistance (HOMA-IR) was calculated from FBS and immunoreactive insulin values; HOMA-IR $=(\mathrm{immu}-$ noreactive insulin $\times$ FBS)/405 [26].

\section{Statistical analysis and software}

The mean and SD values of each item were expressed as mean \pm SD. Shapiro-Wilk test was used as the normality test. If the item showed a normal distribution, one-way analysis of variance (ANOVA) and Tukey's honestly significant difference (HSD) tests were used as parametric tests. In the event that the item did not have a normal distribution, the KruskalWallis $\mathrm{H}$ test and Scheffe test were used as nonparametric tests. We used SPSS15.0J Base System SC Kitsoftware and set the significance level at $\mathrm{p}<0.05$.

\section{Ethical concerns}

Prior to enrollment, subjects were informed about the purpose and method of this study, following which they asked to sign written informed consent forms. If the subjects were minors, we obtained their parents' approval. We ensured that no one of the subjects would be pressured or harmed because of nonparticipation. Personal data were strictly monitored to maintain confidentiality and to protect the privacy of subjects. This study was approved by the Kurume University Mii Campus Ethical Review Board.

\section{RESULTS}

\section{Characteristics of each type of sleep habits}

Figure 1 illustrates the four categories of sleep habits with the mean and SD of each scale score. The first type was the most regular and sound sleep, but there was a slight tendency towards shorter sleep duration. It was termed the "good sleep" type. The second type was somewhat regular and characterized by insomnia and longer sleeping period. It was called "long sleep" type. The third type was somewhat irregular but sound sleep and was characterized by the shortest sleep duration among all subjects. It was called "short sleep" type. The fourth type was the most irregular and was characterized by extreme insomnia and long sleep duration. It was referred to as the "poor sleep" type.

The upper section of Table 2 lists the averages for regular sleep duration, ideal sleep duration, regular bedtime, regular wake-up time, irregular sleep duration, irregular bedtime, irregular wake-up time and time to fall asleep for the four sleep habits groups. Regular sleep duration averaged $6.7 \pm 1.2 \mathrm{~h}$. There were sig- 


\section{Good sleep}

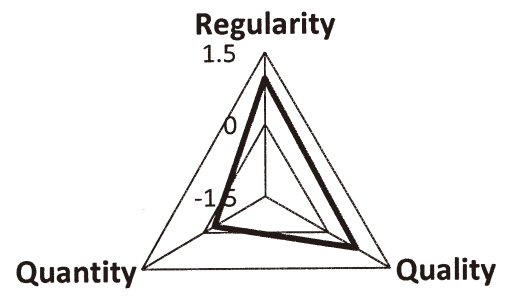

$\mathrm{n}=25$

related Sleep Regularity: $0.959 \pm 0.603$

related Sleep Quality: $0.673 \pm 0.807$

related Sleep Quantity: $-0.266 \pm 0.542$

Total score: $1.367 \pm 1.057$

\section{Short sleep}

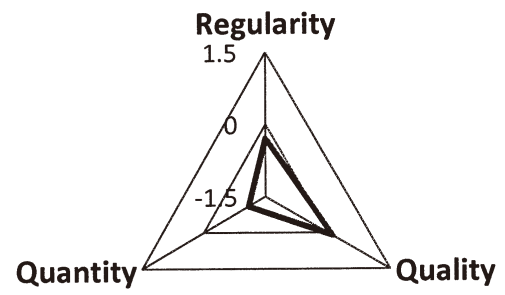

$$
\mathrm{n}=20
$$

related Sleep Regularity: $-0.287 \pm 0.567$ related Sleep Quality: $0.117 \pm 0.472$

related Sleep Quantity: $-1.086 \pm 0.702$

Total score: $-1.256 \pm 0.663$

\section{Long sleep}

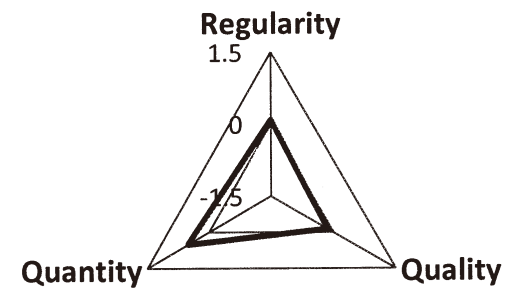

$\mathrm{n}=31$

related Sleep Regularity: $0.094 \pm 0.589$

related Sleep Quality: $-0.145 \pm 1.059$

related Sleep Quantity: $0.514 \pm 0.607$

Total score: $0.463 \pm 1.142$

\section{Poor sleep}

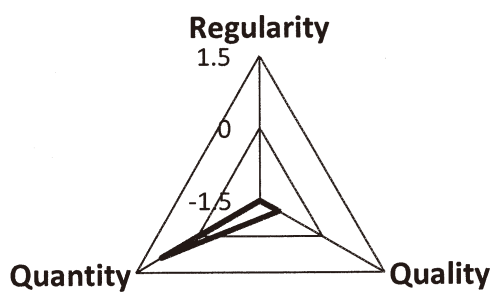

$\mathrm{n}=14$

related Sleep Regularity: $-1.511 \pm 0.727$

related Sleep Quality: $-1.048 \pm 0.779$

related Sleep Quantity: $0.887 \pm 1.143$

Total score: $-1.671 \pm 1.321$

Fig. 1. Four types of sleep habits and corresponding scale scores (means \pm SD).

Good sleep was the most regular and sound sleep, but there was a slight tendency towards shorter sleep duration. Long sleep was somewhat regular and characterized by insomnia and longer sleeping period. Short sleep was somewhat irregular but sound sleep and was characterized by the shortest sleep duration. Poor sleep was the most irregular and was characterized by extreme insomnia and long sleep duration.

nificant differences in regular sleep duration (multiple comparison; short sleep vs. good sleep, long sleep and poor sleep: $\mathrm{p}<0.001$ ), ideal sleep duration (short sleep vs. long sleep: $\mathrm{p}<0.05$ and poor sleep: $\mathrm{p}<0.01)$, regular bedtime (good sleep vs. long sleep: $\mathrm{p}<0.01$, short sleep and poor sleep: $\mathrm{p}<0.001$, long sleep vs. poor sleep: $\mathrm{p}<0.001$, short sleep vs. poor sleep: $\mathrm{p}<0.05)$, regular wake-up time (good sleep vs. long sleep and poor sleep: $\mathrm{p}<0.001$, long sleep vs. short sleep: $\mathrm{p}<0.05$ and poor sleep: $\mathrm{p}<0.001$, short sleep vs. poor sleep: $\mathrm{p}<0001$ ), irregular sleep duration (good sleep vs. short sleep: $p<0.05$ and poor sleep: $p<0.001$, long sleep vs. poor sleep: $\mathrm{p}<0.001$, short sleep vs. poor sleep: $\mathrm{p}<0.001$ ), irregular bedtime (good sleep vs. short sleep: $\mathrm{p}<0.01$ and poor sleep: $\mathrm{p}<0.001$, long sleep vs. poor sleep: $\mathrm{p}<0.001$, short sleep vs. poor sleep: $\mathrm{p}<0.05$ ), irregular wake-up time (good sleep vs. short sleep: $p<0.05$ and poor sleep: $p<0.001$, long sleep vs. poor sleep: $\mathrm{p}<0.001$, short sleep vs. poor sleep: $\mathrm{p}<0.001$ ), and time to fall asleep (good sleep vs. poor sleep: $\mathrm{p}<.001$, short sleep vs. poor sleep: $\mathrm{p}<0.01$ ).

\section{Physical characteristics of subjects}

The middle section of Table 2 describes the physi- 
TABLE 2.

Characteristics of each type of sleep habits (mean $\pm S D$ )

\begin{tabular}{|c|c|c|c|c|c|c|c|}
\hline & & Total & Good sleep & Long sleep & Short sleep & Poor sleep & $\mathrm{p}$ value \\
\hline \multirow{9}{*}{$\begin{array}{l}\text { Sleep } \\
\text { data }\end{array}$} & $\mathrm{n}$ & 90 & 25 & 31 & 20 & 14 & \\
\hline & regular sleep duration (h) & $6.7 \pm 1.2$ & $6.7 \pm 0.8$ & $7.3 \pm 0.7$ & $5.3 \pm 0.9$ & $7.1 \pm 1.5$ & $<0.001^{*}$ \\
\hline & ideal sleep duration (h) & $7.4 \pm 1.2$ & $7.3 \pm 0.9$ & $7.7 \pm 1.1$ & $6.8 \pm 0.7$ & $8.1 \pm 1.7$ & $0.003 *$ \\
\hline & regular bedtime (o’ clock) & $24.7 \pm 1.3$ & $23.6 \pm 1.1$ & $24.6 \pm 0.9$ & $25.2 \pm 1.0$ & $26.1 \pm 1.1$ & $<0.001 *$ \\
\hline & regular wake-up time (o' clock) & $7.8 \pm 1.4$ & $6.7 \pm 0.9$ & $8.2 \pm 0.9$ & $7.4 \pm 0.9$ & $9.6 \pm 1.3$ & $<0.001 *$ \\
\hline & irregular sleep duration (min) & $71.0 \pm 62.9$ & $32.4 \pm 35.6$ & $60.0 \pm 49.6$ & $79.5 \pm 53.6$ & $152.1 \pm 67.1$ & $<0.001 *$ \\
\hline & irregular bedtime (min) & $94.0 \pm 73.6$ & $50.0 \pm 25.8$ & $78.7 \pm 42.3$ & $117.0 \pm 79.6$ & $173.6 \pm 102.7$ & $<0.001^{*}$ \\
\hline & irregular wake-up time (min) & $81.6 \pm 74.1$ & $41.2 \pm 31.0$ & $62.3 \pm 37.9$ & $90.0 \pm 66.0$ & $184.3 \pm 103.2$ & $<0.001 *$ \\
\hline & time to fall asleep (min) & $25.3 \pm 16.4$ & $17.8 \pm 9.3$ & $28.1 \pm 17.9$ & $21.0 \pm 11.1$ & $38.6 \pm 20.3$ & $<0.001 *$ \\
\hline \multirow{6}{*}{$\begin{array}{l}\text { Physical } \\
\text { data }\end{array}$} & Age (years) & $19.4 \pm 1.8$ & $19.0 \pm 0.9$ & $19.6 \pm 2.1$ & $19.2 \pm 2.4$ & $19.9 \pm 1.3$ & n.s. \\
\hline & $\operatorname{BMI}\left(\mathrm{kg} / \mathrm{m}^{2}\right)$ & $21.0 \pm 1.7$ & $20.2 \pm 1.6$ & $21.2 \pm 1.8$ & $21.3 \pm 1.5$ & $21.3 \pm 1.8$ & n.s. \\
\hline & waist circumference $(\mathrm{cm})$ & $72.1 \pm 5.1$ & $69.9 \pm 4.7$ & $72.1 \pm 5.6$ & $73.9 \pm 3.9$ & $73.4 \pm 5.2$ & $0.040 *$ \\
\hline & systolic blood pressure (mmHg) & $116.2 \pm 10.1$ & $110.1 \pm 9.6$ & $117.2 \pm 10.6$ & $118.0 \pm 9.1$ & $120.9 \pm 7.7$ & $0.011^{*}$ \\
\hline & diastolic blood pressure (mmHg) & $66.3 \pm 9.6$ & $62.4 \pm 6.6$ & $68.1 \pm 10.3$ & $65.0 \pm 9.4$ & $70.9 \pm 10.3$ & $0.026 *$ \\
\hline & pulse (/min) & $69.7 \pm 10.3$ & $64.8 \pm 10.5$ & $70.8 \pm 10.3$ & $71.9 \pm 10.2$ & $72.7 \pm 7.6$ & $0.038 *$ \\
\hline \multirow{8}{*}{$\begin{array}{l}\text { Blood } \\
\text { test data }\end{array}$} & FBS (mg/dl) & $86.8 \pm 7.2$ & $85.9 \pm 5.0$ & $86.1 \pm 5.2$ & $85.7 \pm 4.5$ & $91.7 \pm 13.6$ & $0.049^{*}$ \\
\hline & immunoreactive insulin $(\mu \mathrm{IU} / \mathrm{ml})$ & $6.9 \pm 7.0$ & $5.7 \pm 2.2$ & $6.3 \pm 3.4$ & $6.5 \pm 3.0$ & $11.1 \pm 16.2$ & n.s. \\
\hline & HOMA-IR & $1.6 \pm 2.3$ & $1.2 \pm 0.5$ & $1.4 \pm 0.7$ & $1.4 \pm 0.7$ & $3.0 \pm 5.5$ & n.s. \\
\hline & HDL-C (mg/dl) & $61.2 \pm 10.0$ & $63.5 \pm 9.5$ & $57.7 \pm 8.4$ & $65.0 \pm 11.8$ & $59.3 \pm 9.3$ & $0.035^{*}$ \\
\hline & LDL-C (mg/dl) & $90.2 \pm 19.3$ & $93.7 \pm 20.0$ & $87.1 \pm 18.4$ & $85.9 \pm 15.3$ & $97.2 \pm 23.4$ & n.s. \\
\hline & leptin $(\mathrm{ng} / \mathrm{ml})$ & $2.4 \pm 1.1$ & $2.0 \pm 0.8$ & $2.5 \pm 1.3$ & $2.7 \pm 1.0$ & $2.4 \pm 1.0$ & $0.024^{\dagger}$ \\
\hline & desacyl-ghrelin (fmol/ml) & $203.7 \pm 99.6$ & $240.6 \pm 129.5$ & $182.5 \pm 75.3$ & $181.4 \pm 88.6$ & $216.4 \pm 88.5$ & n.s. \\
\hline & HMW-adiponectin $(\mu \mathrm{g} / \mathrm{ml})$ & $5.2 \pm 2.7$ & $5.9 \pm 2.7$ & $5.1 \pm 2.8$ & $4.9 \pm 2.7$ & $4.6 \pm 2.5$ & n.s. \\
\hline
\end{tabular}

* significant difference (by ANOVA test) ${ }^{\dagger}{ }^{\dagger}$ significant difference (by Kruskal-Wallis H test), n.s = not significant.

cal characteristics of subjects associated with the four types of sleep habits. There were significant differences in waist circumference (multiple comparison; good sleep vs. short sleep: $\mathrm{p}<0.05$ ), systolic blood pressure (good sleep vs. poor sleep: $p<0.05$ ), diastolic blood pressure (good sleep vs. poor sleep: $p<0.05$ ), and pulse (no difference in multiple comparison). However, no significant differences were observed in age or BMI among the subjects.

\section{Mental health of subjects}

Figure 2 shows data for the four types of sleep habits based on responses to PCS and MCS of SF-36. While PCS scores of all groups were higher than the national standard value $(\geqq 50)$, MCS scores of two groups (i.e., long sleep and poor sleep) were lower than the national average. There was a significant difference in MCS but no significant difference in PCS among the subjects. Scores for poor sleep were significantly lower than for the other types of sleep with regard to MCS (vs. good sleep: $p<0.001$, vs. long sleep: $p<0.05$, vs. short sleep: $p<0.01$ ). Figure 3 shows data for the four types of sleep habits based on responses to SDS. Scores for poor sleep were significantly higher than those for the other types of sleep (vs. good sleep: $\mathrm{p}<0.001$, vs. long sleep: $\mathrm{p}<0.05$, vs. short sleep: $\mathrm{p}<0.05$ ).

\section{Blood test results}

The bottom section of Table 2 lists the results of blood tests for each group. There were significant differences in FBS (no difference in multiple comparison), HDL-C (long sleep vs. short sleep: $\mathrm{p}<0.05$ ), and leptin (good sleep vs. short sleep: $p<0.05$ ), but no significant differences in immunoreactive insulin levels, HOMA-IR, LDL-C, des-acyl ghrelin, and HMW- 


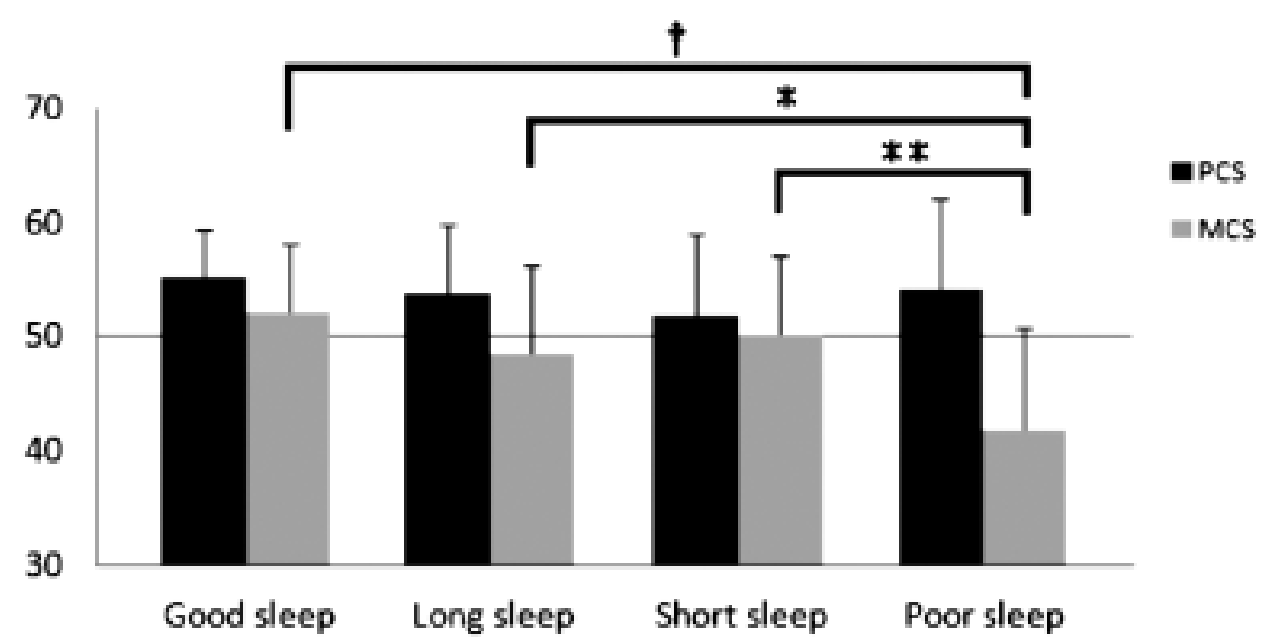

Fig. 2. PCS and MCS as measured by SF-36 (national standard value $=50, * \mathrm{p}<0.05$, ** $\mathrm{p}<0.01,{ }^{\dagger} \mathrm{p}<0.001$ by Tukey's HSD test).

While PCS scores of all groups were higher than national standard value $(\geqq 50)$, MCS scores of long sleep and poor sleep were lower than it. Scores for poor sleep were significantly lower than for the other types of sleep with regard to MCS.

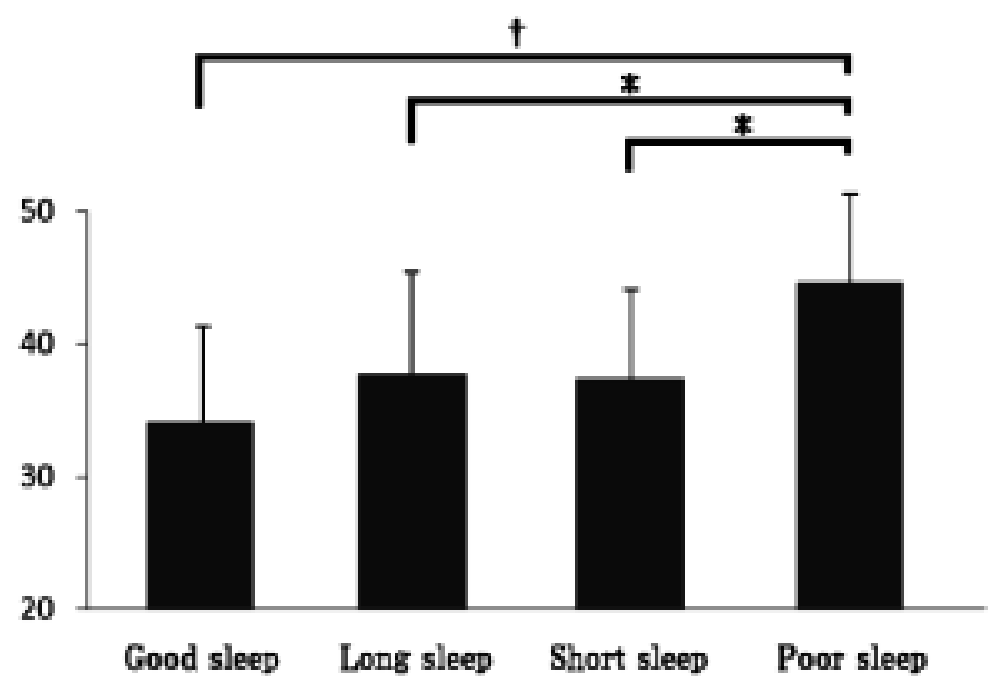

Fig. 2. SDS of four sleep habits $\left(* \mathrm{p}<0.05,{ }^{\dagger} \mathrm{p}<0.001\right.$ by Tukey’s HSD test).

Poor sleep had significantly higher score when compared to the scores for the other types.

adiponectin among the subjects.

\section{DISCUSSION}

\section{Methodology}

The purpose of this study was to measure the impact of different sleep habits (sleep hygiene) on physical and mental health in a cohort of 90 male university students by classifying sleep habits. We developed comprehensive scales that described sleep regularity, quality, and quantity by factor analysis and principal component analysis. Cluster analysis was then employed to classify the sleep habits these students into four groups. Measures of sleep habits must consider not only the number of hours slept (quantity), but must also account for regularity and quality of sleep to 
clearly identify those facets of sleep patterns that most influence mental and physical health. Takeuchi [15] classified sleep habits on the basis of all three factors, and this method proved to be more precise than methods that only evaluated single factors such as sleep duration. We investigated the physical and mental health of the present subjects after classifying sleep habits by means of Takeuchi's comprehensive scoring method. Our analysis underscores the importance of measuring all three dimensions to establish a consistent relation between sleep habits and physical and mental health.

\section{Interpretation of each type of sleep habits}

Good sleep is defined by consistent and normal sleep duration. Those subjects in the good sleep group kept very early hours and scored higher in Morningness. Daily changes of sleep duration, bedtime, and wakeup time were quite small. They also fell asleep easily. Good sleep is the exemplary sleeping pattern. In long sleep subjects, regular bedtimes were normal and regular, but regular wake-up time was later. In addition, both usual and ideal sleep durations were longer than the average. In other words, this group preferred to rise later. They fell asleep with difficulty and had lower quality sleep; that may cause them to oversleep. In the short sleep group, regular bedtimes were later and regular wake-up times were earlier. While this group fell asleep easily, they had irregular sleep habits. They preferred to stay up late and were less concerned about sleep because their ideal sleep duration was shorter than that of any of the other groups. These factors could be expected to result in short sleeping times. Subjects in the poor sleep group seemingly got plenty of sleep according to regular sleep duration, but sleep regularity was poor, and these subjects found it difficult to fall asleep. In addition, their ideal sleep duration was longer than that of any of the other groups. This group may lack sufficient sleep due to chronic insomnia.

In Takeuchi's study [15], several extracted sleep habits were similar to sleep disorders defined by the American Sleep Disorders Association (ASDA), including sleep deficit syndrome, circadian rhythm disorder, and delayed sleep phase syndrome. In the current study, both short and poor sleep subjects had lower than average total scale scores, indicating problematic sleep habits. Short sleep was similar to sleep deficit syndrome as defined by the ASDA in that there was a large gap between actual and ideal sleep durations. In contrast, poor sleep resembled exogenous circadian rhythm disorders such as jet lag. A change in time zone of over three hours causes jet lag with disturbance of sleep induction [27]. The sleep habits of subjects with poor sleep appeared similar to those of daily travelers suffering from jet lag. If the subjects in the poor sleep group graduate from university with the same sleep habits, they might not be able to conform to regular office hours. Thus, they should receive therapy to correct irregular lifestyle habits and to manage insomnia.

\section{Physical characteristics of subjects}

Lack of sleep may be linked to obesity due to increased food consumption [8]. There was a significant difference in average waist circumference between the short and the good sleep groups. However, the good sleep group had the second shortest average sleep duration; thus, shorter sleep duration alone does not lead to weight gain. If individuals continue to sleep for irregular hours and have an inconsistent circadian rhythm, the clock gene begins to express abnormalities, which may lead to easy accumulation of visceral fat $[28,29]$. Therefore, it is suggested that people with irregular as well as short sleep habits, like the short sleep group in this study, may be prone to visceral fat obesity even if their age and BMI are similar to individuals with good sleep habits.

There were significant differences in blood pressure and pulse between the poor and good sleep groups. Ishii [30] demonstrated that irregular sleep habits could disrupt autonomic nervous system function leading to hypertension and rapid pulse. In addition, Javaheri [31] reported that sleep disturbance could cause hypertension even in young healthy men without arteriosclerosis such as the poor sleep subjects in this study. Extremely irregular sleep habits with insomnia, as exhibited by the poor sleep group, may increase blood pressure and pulse due to an autonomic disorder.

\section{Mental health of subjects}

Apropos of the relationship between sleep and mental disease, insomnia and depression are often associated with poor sleep habits. Predictably, poor sleep, the type for which the related Sleep Quality scores were extremely low, indicated the worst mental condition. With respect to MCS measured on the basis of SF-36 and SDS scores, subjects in the poor sleep group had very bad scores that were significantly poorer than the scores of subjects of any other sleep type. SDS scores in this group were $\geqq 40$, which is considered to reflect a state of neurosis. People who have sleep habits like those in the poor sleep group are therefore prone to depression. The mental condition of subjects in the long sleep group, whose related Sleep Quality scores were second-lowest after those in the poor sleep 
group, was also lower than national standard value in MCS in spite of surpassing that of subjects in the short sleep group whose total sleep habits scale score was far worse.

The timing of onset of depression and insomnia has not been sufficiently investigated. However, according to Ohayan [32], insomnia often precedes depression. Furthermore, for people who suffer from insomnia for more than one year, the risk of depression can be 40 times greater than that in those without insomnia [33]. It is important, therefore, that the symptoms observed in the poor sleep group of this study should be immediately resolved, and people whose sleep habits fall into the long sleep category should also be concerned about the relation between their sleep habits and mental condition.

\section{Blood test results}

Of the saccharometabolism items (FBS, immunoreactive insulin, and HOMA-IR), only FBS showed a significant difference among subjects with the four types of sleep habits. However, for all three items, the good sleep group had the lowest values and the poor sleep group showed the highest values. As mentioned above, an inconsistent circadian rhythm because of irregular sleep habits leads to abnormalities in the clock gene, which in turn may lead to visceral fat accumulation $[28,29]$. Eventually, insulin resistance and impaired glucose tolerance can also result.

Of the lipometabolism items (HDL-C and LDL-C), only HDL-C showed a significant difference between long sleep and short sleep. While subjects in the good sleep and short sleep groups had high levels of HDL-C, those in the long sleep and poor sleep groups had low levels of HDL-C. It has been suggested that deterioration in sleep quality and hypersomnia play a role in decreasing HDL-C. Contrary to our result, however, Bjorvatn [34] reported that sleep of short duration decreased HDL-C. This point, therefore, needs further study and consideration.

In the blood tests for leptin, des-acyl ghrelin, and HMW-adiponectin, only leptin showed a significant difference among subjects with the four types of sleep habits. Leptin and des-acyl ghrelin are appestat factors that are influenced by sleep. Sleep of short duration decreases leptin and increases des-acyl ghrelin, which in turn can bring about obesity due to enhanced appetite [35]. We predicted that leptin would decrease and desacyl ghrelin would increase in the short sleep and poor sleep groups. However, there was no difference in desacyl ghrelin, and leptin increased in subjects in the short sleep group. There was a significant difference in circulating leptin between the short and good sleep groups. Leptin levels often increase with obesity because increased visceral fat leads to leptin resistance [36]. Visceral fat accumulation resulting from a chronic lack of sleep and irregular sleep habits might cause leptin resistance. Most previous studies were shortterm (i.e., they analyzed acute sleep insufficiency). The effects of long-term sleep habits have not been adequately considered. Other studies analyzed only sleep duration and not regularity and quality. Therefore, it has been suggested that irregular as well as short sleep habits over the long-term, like short sleep in this study, cause leptin resistance and a compensatory increase in leptin.

In this study, there was no significant difference in HMW-adiponectin between groups. However, HMWadiponectin levels were highest in the good sleep group and lowest in the poor sleep group. Few studies have reported the relationship between adiponectin and sleep. There is a possibility that the small number of subjects in our study prevented us from finding a significant difference for this parameter. Therefore, the relationship between adiponectin and sleep requires further analysis with a larger study sample.

The limitations of this study include the inclusion of males only and the relatively small sample population. Triglyceride, which is one of the diagnostic criteria of metabolic syndrome, was not measured, so we were unable to estimate the relation between sleep habits and metabolic syndrome. We also did not ask about club activities and part-time jobs, factors which could impact regularity of sleep. In addition, TMIN-LHI is a not scale for scoring but a simply questionnaire, and is not subject to validation. Finally, the analyses in this study have a variability related to the subjects. University students have unique lifestyles and sleep habits compared to the general population, and their sleep characteristics may differ from those in heterogeneous subjects like general members of society or the aged.

\section{CONCLUSION}

We found significant differences in certain physical characteristics, mental health, and blood test results among four different sleep habit groups in male university students. Although all subjects in this study were young men with normal BMI, irregular sleep habits caused increases in waist circumference, blood pressure, and pulse, and impaired saccharometabolism and lipometabolism even if sleep duration was kept constant. In addition, the mental condition of subjects with poor quality sleep was not as good as that of sub- 
jects with good quality sleep. It is important to gain a comprehensive understanding of sleep habits in order to maintain good physical and mental health among male university students. Further studies to validate these scales may be required in the fields of sleep medicine and preventive medicine.

\section{REFERENCES}

1. Shiohara A, and Watanabe M. Long-term (5-year) effects of a dietary intervention for reducing the risk factors of metabolic syndrome among Japanese male workers. Jpn J Health \& Human Ecology 2010; 76:131-142.

2. Miyatake N, Matsumoto S, Miyachi M, and Numata T. Increasing oxygen uptake at ventilatory threshold is associated with improving metabolic syndrome in Japanese men. J Prev Med 2009; 4:11-16.

3. Kaji T, Mishima K, Kitamura S, Enomoto M, Nagase Y et al. Relationship between late-life depression and life stressors: Large-scale cross-sectional study of a representative sample of the Japanese general population. Psychiatry Clin Neurosci 2010; 64:426-434.

4. Kohyama J. Suiminnoseiritorinsyou: Shindantochiryosya; 2008 (in Japanese).

5. Asaoka S, Komada Y, Fukuda K, Sugiura T, Inoue Y et al. Exploring the daily associated with delayed bedtime of Japanese university students. Tohoku J Exp Med 2010; 221:245-249.

6. Belenky G, Wesensten NJ, Thorne DR, Thomas ML, Sing $\mathrm{HC}$ et al. Patterns of performance degradation and restoration during sleep restriction and subsequent recovery: a sleep dose-response study. J Sleep Res 2003; 12:1-12.

7. Kaneita Y, Ohida T, Uchiyama M, Takemura S, Kawahara $\mathrm{K}$ et al. The relationship between depression and sleep disturbances: a Japanese nationwide general population survey. J Clin Psychiatry 2006; 67:196-203.

8. Gangwisch JE, Malaspina D, Boden-Albala B, and Heymsfield SB. Inadequate sleep as a risk factor for obesity: Analyses of the NHANESI. Sleep 2005; 28:1289-1296.

9. Tochikubo O, Ikeda A, Miyajima E, and Ishii M. Effects of insufficient sleep on blood pressure monitored by a new multibiomedical recorder. Hypertension 1996; 27:13181324.

10. Knutsona LK, Spiegel K, Penev P, and Cauter EV. The metabolic consequences of sleep deprivation. Sleep Med Rev 2007; 11:163.

11. Kang J, and Chen S. Effects of an irregular bedtime schedule on sleep quality, daytime sleepiness, and fatigue among university students in Taiwan. BMC Public Health 2009; 9:248.

12. Buboltz WC, Franklin B, and Barlow S. Sleep habits and patterns of college students: A preliminary study. J Am Coll Health 2001; 50:131-135.

13. Chang PP, Ford DE, Mead LA, Cooper-Patrick L, and Klag MJ. Insomnia in young men and subsequent depression: The Johns Hopkins Precursors Study. Am J Epidemiol 1997; 146:105-114.

14. Hayashi M, and Hori T. Survey on a sleep habits for university and high school students. Mem Fac Intgr Arts Sci
Hiroshima Univ 1987; 11:53-63. (in Japanese).

15. Takeuchi $T$, Inugami $M$, Ishihara $K$, and Fukuda $K$. Construction of Sleep-Hygiene Scales and Classification of Sleep Patterns in Undergraduates. Jpn J Educ Psychol 2000; 48:294-305. (in Japanese).

16. Matsumoto H, Ogawara K, Kimura H, Amano S, Chinen Y et al. Investigation of lifestyle and mental health in Tokai University students. Bull Fac Phys Educ Tokai Univ 2011; 40:165-171. (in Japanese).

17. Mniszek DH. Bright sleep survey: A study of sleep in 20-45-year olds. J Int Med Res 1988; 16:61-65.

18. Horne JA, and Ostberg O. A self-assessment questionnaire to determine Morningness-Eveningness in human circadian rhythms. Int J Chronobiol 1976; 4:97-110.

19. Ishihara K, Miyashita A, Inugami M, Fukuda K, Yamazaki $\mathrm{K}$ et al. The results of investigation by the Japanese version of Morningness-Eveningness Questionnaire. Jpn J Psychol 1986; 57:87-91.

20. Fukuhara S, Bito S, Green J, Hsiao A, and Kurokawa K. Translation, adaptation, and validation of the SF-36 Health Survey for use in Japan. J Clin Epidemiol 1998; 51:10371044.

21. Fukuhara S, Ware JE, Kosinski M, Wada S, and Gandek B. Psychometric and clinical tests of validity of the Japanese SF-36 Health Survey. J Clin Epidemiol 1998; 51: 1045 1053.

22. Fukuhara S, and Suzukamo Y. Manual of SF-36v2 Japanese version: Institute for Health Outcomes \& Process Evaluation Research Kyoto: 2004 (in Japanese).

23. Fukuda K, and Kobayashi S. A study on a Self-Rating Depression Scale. Psychiatria et Neurologia Japonica 1973; 75:673-679. (in Japanese).

24. Zung WWK. A self-rating depression scale. Arch Gen Psychiat 1965; 12:63-70.

25. Zung WWK, Richards CB, Gables C, and Short MJ. Selfrating depression scale in an outpatient clinic. Arch Gen Psychiat 1965; 13:508-515.

26. Matthews DR, Hosker JP, Rudenski AS, Naylor BA, Treacher DF, et al. Homeostasis model assessment: insulin resistance and $\beta$-cell function from fasting plasma glucose and insulin concentrations in man. Diabetologia 1985; 28:412-419.

27. Rose D, Jung D, Parera D, and Konietzko J. Time-zone shift and jet lag after long-distance flight. ZaeFQ 1999; 93:485-490. (in German).

28. Shimba S. Examine the relativity of the body clock and obesity. Vasc Med 2009; 5:290-294. (in Japanese).

29. Turek FW, Joshu C, Kohsaka A, Lin E, Ivanova G et al. Obesity and metabolic syndrome in circadian Clock mutant mice. Science 2005; 308:1043-1045.

30. Ishii N, Iwata T, Dakeishi M, and Murata K. Effects of shift work on autonomic and neuromotor functions in female nurses. J Occup Health 2004; 46:352-358.

31. Javaheri S, Storfer-Isser A, Rosen CL, and Redline S. Sleep quality and elevated blood pressure in adolescents. Circulation 2008; 118:1034-1040.

32. Ohayon MM, and Roth T. Place of chronic insomnia in the course of depressive and anxiety disorders. J Psychiatr Res 2003; 37:9-15.

33. Ford DE, and Kamerow DB. Epidemiologic study of sleep 
disturbances and psychiatric disorders: An opportunity for prevention. J Am Med Assoc 1989; 262:1479-1484.

34. Bjorvatn B, Sagen IM, Oyane N, Waage S, and Fetveit A. The association between sleep duration, body mass index and metabolic measures in the Hordaland Health Study. Sleep Res 2007; 16:66-76.

35. Spiegel K, Tasali E, Penev P, and Cauter EV. Brief commu- nication: Sleep curtailment in healthy young men is associated with decreased leptin levels, elevated ghrelin levels, and increased hunger and appetite. Ann Intern Med 2004; 141:846-850.

36. Shimizu H, Oh-i S, Okada S, and Mori M. Leptin resistance and obesity. Endocr J 2007; 54:17-26. 